\title{
THE ORDER CONVERGENCE OF MARTINGALES INDEXED BY DIRECTED SETS
}

BY

\author{
KENNETH A. ASTBURY ${ }^{1}$
}

\begin{abstract}
We obtain a condition on the underlying family of $\sigma$-algebras which is properly weaker than the Vitali property but which is also a sufficient condition for the order convergence of martingales of semibounded variation. We also obtain a sufficient condition for the order convergence of martingales of semibounded variation in terms of the finiteness of the extreme order limits of martingales of bounded variation.
\end{abstract}

1. Introduction. Let $(E, \mathscr{B}, \mu)$ be a $\sigma$-finite measure space and let $\theta$ be a directed set under the relation $\ll .(\theta$ is nonempty, and $\ll$ is reflexive, transitive, and has the following property: for each $\rho, \sigma \in \theta$ there exists $\tau \in \theta$ such that $\tau \gg \rho, \tau \gg \sigma$.) Let $\left(\mathscr{B}_{\tau}\right)_{\tau \in \theta}$ be a family of sub- $\sigma$-algebras of $\mathscr{B}$ such that $\left(E, \mathscr{B}_{\tau}, \mu\right)$ is $\sigma$-finite for each $\tau \in \theta$, and $\mathscr{B}_{\rho} \subseteq \mathscr{G}_{\sigma}$ for each $\rho \ll \sigma$. By function we mean an extended real-valued, $\mathscr{B}$-measurable function. Throughout this paper, sets and functions are considered equal if they are equal except on a $\mu$-nullset. Consequently, for $B \in \mathscr{B}$, $\mu(B)=0$ if and only if $B=\varnothing$. Our setting and notation are virtually the same as those of [2].

We recall that the essential supremum and essential infimum of a family of functions $\left(f_{\tau}\right)_{\tau \in \theta}$ are the unique functions ess $\sup _{\tau \in \theta} f_{\tau}$ and ess $\inf _{\tau \in \theta} f_{\tau}$ such that all functions $g$ satisfy:

(i) $f_{\tau} \leqslant g$ for all $\tau \in \theta \Leftrightarrow$ ess $\sup _{\tau \in \theta} f_{\tau} \leqslant g$;

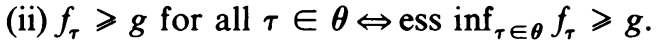

For a family of functions $\left(f_{\tau}\right)_{\tau \in \theta}$, the extreme order limits are defined by

$$
\begin{aligned}
& \underset{\tau \in \boldsymbol{\theta}}{\lim \sup } f_{\tau}=\underset{\rho \in \boldsymbol{\theta}}{\operatorname{ess} \inf }\left(\underset{\tau \gg \rho}{\operatorname{ess} \sup } f_{\tau}\right) \text {, } \\
& \underset{\tau \in \theta}{\lim \inf } f_{\tau}=\underset{\rho \in \theta}{\operatorname{ess} \sup }\left(\underset{\tau \gg \rho}{\text { ess inf }} f_{\tau}\right)
\end{aligned}
$$

[3]. Whenever the extreme order limits are equal, the common function is called the order limit, denoted by $\lim _{\tau \in \theta} f_{\tau}$, and the family $\left(f_{\tau}\right)_{\tau \in \theta}$ is said to be order convergent. For a family of $\mathscr{B}$-members $\left(B_{\tau}\right)_{\tau \in \theta}$, the essential union, essential intersection, extreme order limits, and order convergence are defined analogously.

The conditional expectation of a function $f$ with respect to the sub- $\sigma$-algebra $e$ of $\mathscr{B}$ is denoted by $\mathcal{E}(f \mid \mathcal{C})$. A family of functions $\left(f_{\tau}\right)_{\tau \in \theta}$ is called a martingale whenever $f_{\tau}$ is $\mathscr{B}_{\tau}$-measurable for each $\tau \in \boldsymbol{\theta}$ and $\mathcal{E}\left(f_{\sigma} \mid \mathscr{B}_{\tau}\right)=f_{\tau}$ for each $\sigma \gg \tau$. A

Received by the editors February 26, 1979.

1980 Mathematics Subject Classification. Primary 60G42, 60G44, 60G46; Secondary 60F15, 60G48.

${ }^{1}$ Partially supported by a Wayne State University Faculty Research Award. 
martingale $\left(f_{\tau}\right)_{\tau \in \theta}$ is said to be of bounded (semibounded) variation whenever the set $\left\{\int_{B} f_{\tau} d \mu \mid \tau \in \theta, B \in \mathscr{B}_{\tau}\right\}$ is bounded from above and below (either from above or below).

We call a family of $\mathscr{B}$-members $\left(K_{\tau}\right)_{\tau \in \theta}$ a fine covering whenever $K_{\tau} \in \mathscr{B}_{\tau}$ for each $\tau \in \theta$. For $A \in \mathscr{B}$, the fine covering $\left(K_{\tau}\right)_{\tau \in \theta}$ is called a fine covering of $A$ whenever $\lim \sup _{\tau \in \theta} K_{\tau} \supseteq A$. For $L \in \mathscr{B}$, we denote by $1_{L}$ the indicator function of $L$, which takes the value 1 on $L$, and 0 elsewhere. For a finite sequence of $\Re$-members $\mathcal{L}=\left(L_{1}, L_{2}, \ldots, L_{r}\right)$ we define $\cup \mathcal{L}=\cup_{i=1}^{r} L_{i}$ and $\Sigma \mathcal{L}=$ $\sum_{i=1}^{r} 1_{L_{i}}$

The family $\left(\mathscr{B}_{\tau}\right)_{\tau \in \theta}$ is said to possess the Vitali property whenever the following condition holds: For each $A \in \mathscr{B}$ with $\mu(A)<\infty$, for each fine covering $\left(K_{\tau}\right)_{\tau \in \theta}$ of $A$, and for each $\varepsilon>0$, there exist a finite sequence of $\theta$-members $\left(\xi_{1}, \xi_{2}, \ldots, \xi_{r}\right)$ and a finite sequence of $\mathscr{B}$-members $\mathcal{L}=\left(L_{1}, L_{2}, \ldots, L_{r}\right)$ such that

$$
\begin{aligned}
& L_{i} \in \mathscr{B}_{\xi} \text { and } L_{i} \subseteq K_{\xi_{i}} \text { for each } i=1,2, \ldots, r ; \\
& L_{i} \cap L_{j}=\varnothing \quad \text { for } i \neq j ; \text { and } \mu(A \backslash \cup \mathcal{E})<\varepsilon .
\end{aligned}
$$

(See [2].) We remark that the family $\left(\mathscr{B}_{\tau}\right)_{\tau \in \theta}$ possesses the Vitali property if $\theta$ is totally ordered by $\ll$.

A classical theorem of Krickeberg [3] states the following: If the family $\left(\mathscr{B}_{\tau}\right)_{\tau \in \theta}$ possesses the Vitali property, then every martingale of semibounded variation is order convergent. Although the converse was not expected to be true, the known conditions on the family $\left(\mathscr{B}_{\tau}\right)_{\tau \in \theta}$ which are weaker than the Vitali property imply only that properly smaller classes of martingales are order convergent. (See [2].)

We now introduce the dominated sums property. We denote by $\|\cdot\|_{1}$ and $\|\cdot\|_{\infty}$ the usual norms of $L_{1}(E, \mathscr{B}, \mu)$ and $L_{\infty}(E, \mathscr{B}, \mu)$, respectively. We say that the fine covering $\left(K_{\tau}\right)_{\tau \in \theta}$ satisfies the dominated sums condition whenever the following holds: For some $\delta>0$ and for each $\sigma \in \theta$ there exist a finite sequence of $\theta$-members $\left(\xi_{1}, \xi_{2}, \ldots, \xi_{r}\right)$ and a finite sequence of $\mathscr{B}$-members $\mathcal{E}=$ $\left(L_{1}, L_{2}, \ldots, L_{r}\right)$ such that

$$
\begin{aligned}
& L_{i} \in \mathscr{B}_{\xi}, \quad L_{i} \subseteq K_{\xi} \text { and } \xi_{i} \gg \sigma \text { for each } i=1,2, \ldots, r ; \\
& \|\Sigma \mathcal{E}\|_{1}>\delta\|\Sigma \mathcal{E}\|_{\infty} .
\end{aligned}
$$

We say that the family $\left(\mathscr{B}_{\tau}\right)_{\tau \in \theta}$ possesses the dominated sums property whenever every fine covering $\left(K_{\tau}\right)_{\tau \in \theta}$ with lim $\sup _{\tau \in \theta} K_{\tau} \neq \varnothing$ satisfies the dominated sums condition.

In $\$ 2$ we show that the dominated sums property is properly weaker than the Vitali property. In fact, for each positive integer $n$, the condition obtained by replacing the limitation " $L_{i} \cap L_{j}=\varnothing$ for $i \neq j$ " by " $\|\Sigma \mathcal{L}\|_{\infty} \leqslant n$ " in the statement of the Vitali property is shown to be properly stronger than the dominated sums property. In $\$ 3$ we develop the underlying martingale properties which are used in the following sections. In $\$ 4$ we strengthen Krickeberg's theorem: If the family $\left(\mathscr{B}_{\tau}\right)_{\tau \in \theta}$ possesses the dominated sums property, then every martingale of semibounded variation is order convergent. In $\$ 5$ we establish some decompositions of $E$ in terms of order convergence. 
2. The dominated sums property. The following lemma is a generalization of a well-known property of equivalent measures, and is used in our proof that the dominated sums property is independent of the base measure $\mu$.

LEMMA 2.1. Let $\nu$ be a positive $\sigma$-finite measure on $(E, \mathscr{B})$ equivalent to $\mu$. Let $C \in \mathscr{B}$ be such that $\nu(C)<\infty$. Then for each $\varepsilon>0$ there exists $\delta>0$ such that any finite sequence of $\Re$-members $\mathcal{L}=\left(L_{1}, L_{2}, \ldots, L_{r}\right)$ satisfies

$$
\cup \mathcal{L} \subseteq C, \quad\left\|\sum \mathcal{L}\right\|_{1} \leqslant \delta\left\|\sum \mathcal{L}\right\|_{\infty} \Rightarrow \int \sum \mathcal{L} d \nu \leqslant \varepsilon\left\|\sum \mathcal{L}\right\|_{\infty} .
$$

Proof. Let $f$ be the Radon-Nikodym derivative of $\nu$ with respect to $\mu$. The lemma is valid if $f$ is bounded on $C$, and we treat the other case. Let $\varepsilon>0$. By the Lebesgue Convergence Theorem, there exists a positive integer $n$ such that $\int_{C \cap\{f>n\}} f d \mu<\varepsilon$. Set $\delta=\mu(C \cap\{f>n\}) ; \delta>0$ because $f$ is unbounded on $C$. Let $\mathcal{E}=\left(L_{1}, L_{2}, \ldots, L_{r}\right)$ be a finite sequence of $\mathscr{B}$-members satisfying $\cup \mathcal{E} \subseteq C$ and $\|\Sigma \mathcal{L}\|_{1} \leqslant \delta\|\Sigma \mathcal{L}\|_{\infty}$. Define $\mathfrak{N}$ to be the sequence $(C \cap\{f>n\}, C \cap\{f>$ $n\}, \ldots, C \cap\{f>n\})$ having $\|\mathscr{l}\|_{\infty}$ terms. Then

$$
\left\|\sum \Re\right\|_{1}=\mu(C \cap\{f>n\})\|\mathcal{L}\|_{\infty}=\delta\|\mathcal{L}\|_{\infty} \geqslant\|\mathcal{L}\|_{1}
$$

Furthermore,

$$
\begin{aligned}
\sum \mathfrak{K}-1_{\{f>n\}} \sum \mathcal{L} & \geqslant 0 & \text { on }\{f>n\} \\
& =0 & \text { elsewhere. }
\end{aligned}
$$

Hence,

$$
\begin{aligned}
\int\left(\sum \Re-1_{\{f>n\}}\right. & \left.\sum \mathcal{L}\right) f d \mu \geqslant n \int \sum \Re-1_{\{f>n\}} \sum \mathcal{E} d \mu \\
& \geqslant n \int \sum \mathcal{L}-1_{\{f>n\}} \sum \mathcal{L} d \mu \geqslant \int\left(\sum \mathcal{L}-1_{\{f>n\}} \sum \mathcal{L}\right) f d \mu,
\end{aligned}
$$

and $\int(\Sigma \Re) f d \mu \geqslant \int(\Sigma \mathcal{L}) f d \mu$. Finally

$$
\begin{aligned}
\int \sum \mathcal{L} d \nu & =\int\left(\sum \mathcal{L}\right) f d \mu \leqslant \int\left(\sum \mathfrak{N}\right) f d \mu \\
& =\left\|\sum \mathfrak{N}\right\|_{\infty} \int_{C \cap\{f>n\}} f d \mu \leqslant \varepsilon\left\|\sum \mathcal{L}\right\|_{\infty} .
\end{aligned}
$$

THEOREM 2.1. Let $\nu$ be a positive, $\sigma$-finite measure on $(E, \mathscr{B})$ equivalent to $\mu$. Then the family $\left(\mathscr{B}_{\tau}\right)_{\tau \in \theta}$ possesses the dominated sums property if and only if the family $\left(\mathscr{B}_{\tau}\right)_{\tau \in \theta}$ in the underlying measure space $(E, \mathscr{B}, \nu)$ possesses the dominated sums property.

Proof. By symmetry, it suffices to prove one direction. We prove the if direction by contradiction. Assume $\left(\mathscr{B}_{\tau}\right)_{\tau \in \theta}$ does not possess the dominated sums property. Then there exists a fine covering $\left(K_{\tau}\right)_{\tau \in \theta}$ with $\lim \sup _{\tau \in \theta} K_{\tau} \neq \varnothing$ which does not satisfy the dominated sums condition: For each $\delta>0$ there exists $\sigma \in \theta$ such that 
all finite sequences of $\theta$-members $\left(\xi_{1}, \xi_{2}, \ldots, \xi_{r}\right)$ and $\mathscr{B}$-members $\mathcal{E}=$ $\left(L_{1}, L_{2}, \ldots, L_{r}\right)$ satisfy

$$
\begin{aligned}
& L_{i} \in \mathscr{P}_{\xi_{i}}, \quad L_{i} \subseteq K_{\xi_{i}} \text { and } \xi_{i} \gg \sigma \text { for all } i=1,2, \ldots, r \\
& \Rightarrow\|\Sigma \mathscr{e}\|_{1} \leqslant \delta\|\Sigma \mathscr{L}\|_{\infty} .
\end{aligned}
$$

Let $\rho \in \theta$ and $C \in \mathscr{B}_{\rho}$ be such that $\nu(C)<\infty$ and $C \cap \lim \sup _{\tau \in \theta} K_{\tau} \neq \varnothing$. Define the fine covering $\left(J_{\tau}\right)_{\tau \in \theta}$ by

$$
J_{\tau}= \begin{cases}K_{\tau} \cap C & \text { for } \tau \gg \rho, \\ \varnothing & \text { elsewhere. }\end{cases}
$$

Then

$$
\lim \sup J_{\tau \in \theta}=\limsup _{\tau \in \theta}\left(K_{\tau} \cap C\right)=\left(\lim \sup _{\tau \in \theta} K_{\tau}\right) \cap C \neq \varnothing .
$$

Because $J_{\tau} \subseteq K_{\tau}$ for all $\tau \in \theta,\left(J_{\tau}\right)_{\tau \in \theta}$ also does not satisfy the dominated sums condition. By Lemma $2.1,\left(J_{\tau}\right)_{\tau \in \theta}$ does not satisfy the dominated sums condition in the measure space $(E, \mathscr{B}, \nu)$.

We now define a class of properties which are weaker than the Vitali property but which are stronger than the dominated sums property.

Let $n$ be a positive integer. We say that the family $\left(\mathscr{G}_{\tau}\right)_{\tau \in \theta}$ possesses the Vitali property of degree $n$ whenever the following condition holds: For each $A \in \mathscr{B}$ with $\mu(A)<\infty$, for each fine covering $\left(K_{\tau}\right)_{\tau \in \theta}$ of $A$, and for each $\varepsilon>0$, there exist a finite sequence of $\theta$-members $\left(\xi_{1}, \xi_{2}, \ldots, \xi_{r}\right)$ and a finite sequence of $\mathscr{B}$-members $\mathcal{E}=\left(L_{1}, L_{2}, \ldots, L_{r}\right)$ such that

$$
\begin{aligned}
& L_{i} \in \mathscr{B}_{\xi_{i}} \text { and } L_{i} \subseteq K_{\xi_{i}} \text { for each } i=1,2, \ldots, r ; \\
& \|\Sigma \mathcal{L}\|_{\infty} \leqslant n \text {; and }(A \backslash \cup \mathcal{E})<\varepsilon .
\end{aligned}
$$

Clearly for $n>m$, the Vitali property of degree $n$ is weaker than the Vitali property of degree $m$, and the Vitali property of degree 1 is precisely the Vitali property.

Proposition 2.1. Let $n$ be a positive integer and let the family $\left(\mathscr{B}_{\tau}\right)_{\tau \in \theta}$ possess the Vitali property of degree $n$. Then the family $\left(\mathscr{B}_{\tau}\right)_{\tau \in \theta}$ possesses the dominated sums property.

Proof. Let $\left(K_{\tau}\right)_{\tau \in \theta}$ be a fine covering with $\lim \sup _{\tau \in \theta} K_{\tau} \neq \varnothing$. Let $0<\delta<$ $(2 n)^{-1} \mu\left(\lim \sup _{\tau \in \theta} K_{\tau}\right)$. Let $\sigma \in \theta$. Define the fine covering $\left(J_{\tau}\right)_{\tau \in \theta}$ by

$$
J_{\tau}= \begin{cases}K_{\tau} & \text { for } \tau \gg \sigma \\ \varnothing & \text { elsewhere. }\end{cases}
$$

There exists $A \in \mathscr{B}$ such that $2 \delta n \leqslant \mu(A)<\infty$ and $A \subseteq \lim \sup _{\tau \in \theta} K_{\tau}=$ $\lim \sup _{\tau \in \theta} J_{\tau}$. Applying the definition of Vitali property of degree $n$ to $\left(J_{\tau}\right)_{\tau \in \theta}$, there exist a finite sequence of $\theta$-members $\left(\xi_{1}, \xi_{2}, \ldots, \xi_{r}\right)$ and a finite sequence of $\mathscr{B}$-members $\mathcal{L}=\left(L_{1}, L_{2}, \ldots, L_{r}\right)$ such that $L_{i} \in \mathscr{B}_{\xi}$ and $L_{i} \subseteq K_{\xi}$ for $i=$ $1,2, \ldots, r ;\|\Sigma \mathcal{L}\|_{\infty}<n$; and $(A \backslash \cup \mathcal{L})<\frac{1}{2} \mu(A)$. Then

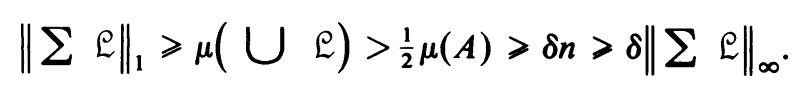


Our next example shows that for each integer $n$ greater than 1 , the Vitali property of degree $n$ is properly weaker than the Vitali property of degree $n-1$.

EXAMPLE 2.1. Let $N$ be the set of positive integers and let $n \in N, n>2$, be fixed. Define the directed set

$$
\begin{aligned}
& \theta=\left\{(i, j) \mid i, j \in N, 1 \leqslant j \leqslant n^{i}\right\} \\
& (i, j) \gg(k, m) \text { if and only if } i>k \text { or }(i, j)=(k, m) .
\end{aligned}
$$

For each fixed $i \in N$ we form $n^{i}$ sets $\left(S_{(i, j)}\right)_{j=1,2, \ldots, n^{i}}$ from a space $U_{i}$ of points as follows: For each group of $n$ of the sets we choose a distinct point and place this point in each set of the group; each set contains only those points inserted under the above procedure. We assume that $U_{i}$ has exactly the number of points required for this construction, namely

$$
\left(\begin{array}{c}
n^{i} \\
n
\end{array}\right)
$$

the combinations of $n^{i}$ things taken $n$ at a time. Let $\mathcal{T}_{i}$ be the set of all subsets of $U_{i}$ and let $P_{i}$ be the uniform probability measure on $\left(U_{i}, \mathcal{T}_{i}\right)$. For each $(i, j) \in \theta$, the number of points in $S_{(i, j)}$ is

$$
\left(\begin{array}{c}
n^{i}-1 \\
n-1
\end{array}\right)
$$

-one point for each group of $n-1$ sets chosen from the other $n^{i}-1$ sets-and

$$
P_{i}\left(S_{(i, j)}\right)=\left(\begin{array}{c}
n^{i}-1 \\
n-1
\end{array}\right) /\left(\begin{array}{c}
n^{i} \\
n
\end{array}\right)=\frac{\left(n^{i}-1\right) ! /\left(n^{i}-n\right) !(n-1) !}{n^{i} ! /\left(n^{i}-n\right) ! n !}=\frac{1}{n^{i-1}} .
$$

Let $\mathcal{S}_{(i, j)}$ be the sub- $\sigma$-algebra of $\mathcal{T}_{i}$ generated by $S_{(i, j)}$ and all singleton sets $\{u\}$ for which $u \notin S_{(i, j)}$, and let $\mathscr{U}_{i}=\left\{\varnothing, U_{i}\right\}$. Define

$$
\begin{aligned}
(E, \mathscr{B}, \mu) & =\prod_{k \in N}\left(U_{k}, \mathscr{T}_{k}, P_{k}\right), \\
\mathscr{B}_{(i, j)} & =\left(\prod_{k=1}^{i-1} \mathcal{T}_{k}\right) \times \mathcal{S}_{(i, j)} \times\left(\prod_{k=i+1}^{\infty} \mathscr{U}_{k}\right), \\
A_{(i, j)} & =\left(\prod_{k=1}^{i-1} U_{k}\right) \times S_{(i, j)} \times\left(\prod_{k=i+1}^{\infty} U_{k}\right) .
\end{aligned}
$$

We remark that $\mu(E)=1$. In order to establish that $\left(\mathscr{B}_{\tau}\right)_{\tau \in \theta}$ possesses the Vitali property of degree $n$, let $A \in \mathscr{B}$, let $\left(K_{\tau}\right)_{\tau \in \theta}$ be a fine covering of $A$, and let $\varepsilon>0$. Then there exists $\sigma \in \theta$ such that $\mu\left(A \backslash \cup_{\tau \ll \sigma} K_{\tau}\right)<\varepsilon$. Define for each $\tau \in \theta$,

$$
M_{\tau}=K_{\tau} \backslash \bigcup_{\substack{\rho \ll \tau \\ \rho \neq \tau}} K_{\rho} \in \Re_{\tau}
$$

We have $\cup_{\tau \ll \sigma} M_{\tau}=\cup_{\tau \ll \sigma} K_{\tau}$, and for $i=1,2, \ldots$ the sets $\cup_{j=1}^{n^{\prime}} M_{(i, j)}$ are disjoint. Fix $i$ and consider for $j=1,2, \ldots, n^{i}$ the partition of $M_{(i, j)}$ :

$$
M_{(i, j)}=\left(M_{(i, j)} \cap A_{(i, j)}\right) \cup\left(M_{(i, j)} \backslash A_{(i, j)}\right) .
$$


We choose a family of sets $\left(L_{(i, j)}\right)_{j=1,2, \ldots, n^{i}}$, each $L_{(i, j)}$ being the union of $\left(M_{(i, j)} \cap A_{(i, j)}\right)$ and a subset of $\left(M_{(i, j)} \backslash A_{(i, j)}\right)$, in such a way that

$$
\begin{gathered}
L_{(i, j)} \backslash A_{(i, j)} \in\left(\prod_{k=1}^{i} \mathcal{T}_{k}\right) \times\left(\prod_{k=i+1}^{\infty} \mathscr{Q}_{k}\right), \\
\bigcup_{j=1}^{n^{i}} L_{(i, j)}=\bigcup_{j=1}^{n^{i}} M_{(i, j)},
\end{gathered}
$$

and for each $1 \leqslant j \leqslant n^{i}$ the two sets $\left(L_{(i, j)} \backslash A_{(i, j)}\right)$ and $\cup_{k=1 ; k \neq j}^{n^{i}} L_{(i, k)}$ are disjoint. We have $L_{(i, j)} \cap A_{(i, j)}=M_{(i, j)} \cap A_{(i, j)} \in \mathscr{B}_{(i, j)}$, and by the definition of $\delta_{(i, j)}$ it follows that $L_{(i, j)} \in \mathscr{B}_{(i, j)}$. Furthermore, by disjointness,

$$
\begin{gathered}
\left\|\sum_{j=1}^{n^{i}} L_{(i, j)}\right\|_{\infty} \leqslant g \operatorname{gmax}\left(\left\|\sum_{j=1}^{n^{i}}\left(L_{(i, j)} \cap A_{(i, j)}\right)\right\|_{\infty}, 1\right) \\
\leqslant \max \left(\left\|\sum_{j=1}^{n^{i}} A_{(i, j)}\right\|_{\infty}, 1\right)=n .
\end{gathered}
$$

Let $\mathcal{E}$ be a sequence of the sets $L_{\tau}, \tau \ll \sigma$. Then

$$
\begin{gathered}
\left\|\sum \mathcal{L}\right\|_{\infty}=\max _{i}\left(\left\|\sum_{j=1}^{n^{i}} L_{(i, j)}\right\|_{\infty}\right)<n, \\
\mu(A \backslash \bigcup \mathcal{L})=\mu\left(A \backslash \bigcup_{\tau \ll \sigma} M_{\tau}\right)=\mu\left(A \backslash \bigcup_{\tau \ll \sigma} K_{\tau}\right)<\varepsilon,
\end{gathered}
$$

and the Vitali property of degree $n$ is established. We now show that the Vitali property of degree $n-1$ does not hold. Let $k \in N$ be such that $\sum_{i-k}^{\infty} 1 / n^{i-2}<\frac{1}{2}$. We denote by $\theta_{i}$ the set of all $\theta$-members whose first coordinate is $i$. Define the fine covering $\left(K_{\tau}\right)_{\tau \in \theta}$ of $E$ by

$$
K_{\tau}= \begin{cases}A_{\tau} & \text { for } \tau \in \bigcup_{i=k}^{\infty} \theta_{i}, \\ \varnothing & \text { elsewhere. }\end{cases}
$$

Let $\left(\xi_{1}, \xi_{2}, \ldots, \xi_{r}\right)$ and $\mathcal{L}=\left(L_{1}, L_{2}, \ldots, L_{r}\right)$ be finite sequences of $\theta$-members and $\mathscr{B}$-members, respectively, such that

$$
\begin{aligned}
& L_{m} \in \mathscr{B}_{\xi_{m}} \text { and } L_{m} \subseteq K_{\xi_{m}} \text { for } m=1,2, \ldots, r \\
& \left\|\sum \mathcal{L}\right\|_{\infty} \leqslant n-1 .
\end{aligned}
$$

Fixing $i>k$ we can write for each $\xi_{m} \in \theta_{i}$,

$$
L_{m}=C_{m} \times S_{\xi_{m}} \times\left(\prod_{p=i+1}^{\infty} U_{p}\right) \quad \text { where } C_{m} \subseteq \prod_{p=1}^{i-1} U_{p}
$$


If $\left\|\sum_{\xi_{m} \in \theta_{i}} C_{m}\right\|_{\infty} \geqslant n$, then $\left\|\sum_{\xi_{m} \in \theta_{i}} L_{m}\right\|_{\infty} \geqslant n$ because every group of $n$ sets from $\left(S_{(i, j)}\right)_{j=1,2, \ldots, n^{i}}$ has a common point; consequently $\left\|\Sigma_{\xi_{m} \in \theta_{i}} C_{m}\right\|_{\infty}<n$. Furthermore

$$
\begin{aligned}
\mu\left(\bigcup_{\xi_{m} \in \theta_{i}} L_{m}\right) & \leqslant \sum_{\xi_{m} \in \theta_{i}} \mu\left(L_{m}\right)=\sum_{\xi_{m} \in \theta_{i}} \mu\left(C_{m} \times \prod_{p=i}^{\infty} U_{p}\right) P_{i}\left(S_{\xi_{m}}\right) \\
& =\frac{1}{n^{i-1}} \sum_{\xi_{m} \in \theta_{i}} \mu\left(C_{m} \times \prod_{p=i}^{\infty} U_{p}\right)=\frac{1}{n^{i-1}}\left\|\sum_{\xi_{m} \in \theta_{i}} C_{m} \times \prod_{p=i}^{\infty} U_{p}\right\|_{1} \\
& \leqslant \frac{1}{n^{i-1}}\left\|\sum_{\xi_{m} \in \theta_{i}} C_{m} \times \prod_{p=i}^{\infty} U_{p}\right\|_{\infty} \mu(E)<\frac{1}{n^{i-2}} .
\end{aligned}
$$

Finally,

$$
\mu(\bigcup \mathcal{L}) \leqslant \sum_{i>k} \mu\left(\bigcup_{\xi_{m} \in \theta_{i}} L_{m}\right)<\sum_{i>k} \frac{1}{n^{i-2}}<\frac{1}{2},
$$

and the Vitali property of degree $n-1$ fails.

3. Countably additive and purely finitely additive martingales. Let $\mathscr{B}_{\infty}$ be the sub- $\sigma$-algebra of $\mathscr{B}$ which is generated by $\cup_{\tau \in \theta} \mathscr{B}_{\tau}$. It is known that for any $A \in \mathscr{B}_{\infty}$ there exists a family of sets $M_{\tau} \in \mathscr{B}_{\tau}$ such that $A=\lim _{\tau \in \theta} M_{\tau}$.

To every martingale of bounded variation $\Phi=\left(f_{\tau}\right)_{\tau \in \theta}$ there corresponds a finitely additive set function (of bounded variation) $Z(\Phi)$ on $\cup_{\tau \in \theta} \Re_{\tau}$ defined by

$$
Z(\Phi)(A)=\int_{A} f_{\tau} d \mu \quad \text { for any } \tau \text { such that } A \in \mathscr{B}_{\tau} .
$$

According to a theorem of Yosida and Hewitt [6], every finitely additive set function can be expressed uniquely as the sum of a countably additive set function and a purely finitely additive set function. A martingale of bounded variation $\Phi$ is called countably additive or purely finitely additive whenever $Z(\Phi)$ is countably additive or purely finitely additive, respectively. It follows that every martingale of bounded variation can be expressed uniquely as the sum of a countably additive martingale and a purely finitely additive martingale. A countably additive set function on $\cup_{\tau \in \theta} \mathscr{B}_{\tau}$ can be extended to a measure on $\left(E, \mathscr{B}_{\infty}\right)$ [1], and by the Radon-Nikodym Theorem, it follows that the countably additive martingales are precisely those of the form $\left(\mathcal{E}\left(f \mid \Re_{\tau}\right)\right)_{\tau \in \theta}$ for some function $f$ with $\int|f| d \mu<\infty$. For a more complete discussion of the above see [2]. The purely finitely additive martingales can be characterized by a well-known property of purely finitely additive set functions [6]: For each $\varepsilon>0$ there exist $\sigma \in \theta$ and $D \in \mathscr{B}_{\sigma}$ such that

(a) $\int\left|1_{D} f_{\tau}\right| d \mu<\varepsilon$ for all $\tau \gg \sigma$, and

(b) $\mu(E \backslash D)<\varepsilon$.

LemMa 3.1. Let $f$ be a function with $\int f d \mu<\infty$, let $a>b>0$, and let $A=$ $\left\{\lim \sup _{\tau \in \theta} \mathcal{E}\left(f \mid \mathscr{G}_{\tau}\right)>a>b \geqslant \mathcal{E}\left(f \mid \mathscr{G}_{\infty}\right)\right\}$. Then there exists a fine covering $\left(K_{\tau}\right)_{\tau \in \theta}$ of $A$ with the following property: For each $\varepsilon>0$ there exist $\sigma \in \theta$ and $C \in \mathscr{B}_{\infty}$ such that

(i) $\int 1_{C} f d \mu<\varepsilon$, and

(ii) $\mathcal{E}\left(1_{C} f \mid \Re_{\tau}\right) \geqslant a-b$ on $K_{\tau}$ for all $\tau \gg \sigma$. 
Proof. $A \in \mathscr{B}_{\infty}$; hence $A=\lim _{\tau \in \theta} M_{\tau}$ for some family of sets $M_{\tau} \in \mathscr{B}_{\tau}$. Define for each $\tau \in \boldsymbol{\theta}$,

$$
K_{\tau}=M_{\tau} \cap\left\{\mathcal{E}\left(f \mid \mathscr{B}_{\tau}\right)>a\right\} \in \mathscr{B}_{\tau} .
$$

Now

$$
\begin{gathered}
\underset{\tau \in \theta}{\lim \sup }\left\{\mathcal{E}\left(f \mid \mathscr{B}_{\tau}\right)>a\right\} \supseteq\left\{\limsup _{\tau \in \theta} \mathcal{E}\left(f \mid \mathscr{B}_{\tau}\right)>a\right\} \supseteq A, \\
\operatorname{lim\operatorname {sup}} K_{\tau}=\left(\lim _{\tau \in \theta} M_{\tau}\right) \cap \underset{\tau \in \theta}{\lim \sup }\left\{\mathcal{E}\left(f \mid \mathscr{B}_{\tau}\right)>a\right\}=A,
\end{gathered}
$$

and $\left(K_{\tau}\right)_{\tau \in \theta}$ is a fine covering of $A$. Let $\varepsilon>0$. For each $\tau \in \theta$ define the set $C_{\tau}=\cup_{\rho \gg \tau}\left(K_{\rho} \backslash A\right)$ and the function

$$
g_{\tau}= \begin{cases}\sup (0, f) & \text { on } C_{\tau}, \\ 0 & \text { elsewhere. }\end{cases}
$$

The family of sets $\left(C_{\tau}\right)_{\tau \in \theta}$ is nonincreasing and

$$
\begin{aligned}
\underset{\tau \in \theta}{\lim \sup } C_{\tau} & =\bigcap_{\tau \in \theta} C_{\tau}=\bigcap_{\tau \in \theta} \bigcup_{\rho \gg \tau}\left(K_{\rho} \backslash A\right)=\lim _{\tau \in \theta} \sup \left(K_{\tau} \backslash A\right) \\
& =\left(\underset{\tau \in \theta}{\lim \sup } K_{\tau}\right) \backslash A=\varnothing ;
\end{aligned}
$$

hence $\lim _{\tau \in \theta} g_{\tau}=0$. By the Lebesgue Convergence Theorem, which is valid also for families of functions indexed by directed sets, there exist $\sigma \in \theta$ and $C=C_{\sigma} \in$ $\mathscr{B}_{\infty}$ such that $\int 1_{C} f d \mu \leqslant \int g_{\sigma} d \mu<\varepsilon$. For $\tau \gg \sigma, L \in \mathscr{B}_{\tau}$ and $L \subseteq K_{\tau}$,

$$
\begin{aligned}
\int_{L} 1_{C} f d \mu & =\int_{L \backslash A} f d \mu=\int_{L} f d \mu-\int_{L \cap A} f d \mu \\
& =\int_{L} \mathcal{E}\left(f \mid \Re_{\tau}\right) d \mu-\int_{L \cap A} \mathcal{E}\left(f \mid \Re_{\infty}\right) d \mu \\
& \geqslant \int_{L} a d \mu-\int_{L \cap A} b d \mu \geqslant \int_{L} a-b d \mu .
\end{aligned}
$$

Hence, for $\tau \gg \sigma, \mathcal{E}\left(1_{C} f \mid \Re_{\tau}\right) \geqslant a-b$ on $K_{\tau}$.

TheOrem 3.1. Let $f$ be a function with $\int f d \mu<\infty$, let $a>b>0$, and let $\varepsilon>0$. Then there exists $C \in \mathscr{B}_{\infty}$ such that

(i) $\int 1_{C} f d \mu<\varepsilon$, and

(ii) $\lim \sup _{\tau \in \theta} \mathcal{E}\left(1_{C} f \mid \Re_{\tau}\right) \geqslant a-b$ on the set $\left\{\lim \sup _{\tau \in \theta} \mathcal{E}\left(f \mid \Re_{\tau}\right)>a>b>\right.$ $\left.\mathcal{E}\left(f \mid \mathscr{B}_{\infty}\right)\right\}$.

Proof. lim $\sup _{\tau \in \theta} \mathcal{E}\left(1_{C} f \mid \mathscr{B}_{\tau}\right) \geqslant a-b$ on the set $\lim \sup _{\tau \in \theta}\left\{\mathcal{E}\left(1_{C} f \mid \mathscr{B}_{\tau}\right)>a-\right.$ b) and apply Lemma 3.1.

LEMMA 3.2. Let $\left(f_{\tau}\right)_{\tau \in \theta}$ be a purely finitely additive martingale, let $a>0$, let $A=\left\{\lim \sup _{\tau \in \theta}\left|f_{\tau}\right|>a\right\}$, and let $\varepsilon>0$. Then there exists a fine covering $\left(K_{\tau}\right)_{\tau \in \theta}$ such that

(i) $\mu\left(A \backslash \lim \sup _{\tau \in \theta} K_{\tau}\right)<\varepsilon$, and

(ii) for each $\gamma>0$ there exist $\sigma \in \theta$ and $C \in \mathscr{B}_{\sigma}$ such that for each $\tau \gg \sigma$, $\left|1_{c} f_{\tau}\right|>a$ on $K_{\tau}$, and $\int\left|1_{C} f_{\tau}\right| d \mu<\gamma$. 
Proof. Let $k$ be a positive integer such that $\Sigma_{n>k} 1 / 2^{n}<\varepsilon$. By the characterization of purely finitely additive martingales given at the beginning of this section, we choose recursively $\sigma_{n} \in \theta$ and $D_{n} \in \mathscr{B}_{\sigma_{n}}$ for $n=k, k+1, \ldots$ satisfying $\int\left|1_{D_{n}} f_{\tau}\right| d \mu<1 / 2^{n}$ for all $\tau \gg \sigma_{n}, \mu\left(E \backslash D_{n}\right)<1 / 2^{n}$, and $\sigma_{n+1} \gg \sigma_{n}$. Define for each $\tau \in \theta$,

$$
K_{\tau}=\left\{\left|f_{\tau}\right|>a\right\} \cap\left(\bigcap_{\sigma_{n} \ll \tau} D_{n}\right) \in \mathscr{B}_{\tau} .
$$

Then

$$
\begin{aligned}
\underset{\tau \in \theta}{\lim \sup } K_{\tau} \supseteq \lim _{\tau \in \theta} \sup \left(\left\{\left|f_{\tau}\right|>a\right\} \cap\left(\bigcap_{n>k} D_{n}\right)\right) \\
=\left(\limsup _{\tau \in \theta}\left\{\left|f_{\tau}\right|>a\right\}\right) \cap\left(\bigcap_{n>k} D_{n}\right) \supseteq A \cap\left(\bigcap_{n>k} D_{n}\right),
\end{aligned}
$$

and

$$
\begin{aligned}
\mu\left(A \backslash \limsup _{\tau \in \theta} K_{\tau}\right) & \left.<\mu\left(A \backslash A \cap\left(\bigcap_{n>k} D_{n}\right)\right]\right)=\mu\left(A \backslash \bigcap_{n>k} D_{n}\right) \\
& \leqslant \mu\left(E \backslash \bigcap_{n>k} D_{n}\right)=\mu\left(\bigcup_{n>k}\left(E \backslash D_{n}\right)\right)<\sum_{n>k} \mu\left(E \backslash D_{n}\right) \\
& <\sum_{n>k} \frac{1}{2^{n}}<\varepsilon .
\end{aligned}
$$

In order to establish (ii), let $\gamma>0$. Choose a positive integer $n$ such that $1 / 2^{n}<\gamma$ and set $\sigma=\sigma_{n} \in \theta, C=D_{n} \in \mathscr{B}_{\sigma}$. Let $\tau \gg \sigma$. Then

$$
\int\left|1_{C} f_{\tau}\right| d \mu=\int\left|1_{D_{n}} f_{\tau}\right| d \mu<1 / 2^{n}<\gamma
$$

furthermore $K_{\tau} \subseteq D_{n}$ and

$$
\left|1_{C} f_{\tau}\right|=\left|1_{D_{n}} f_{\tau}\right|=\left|f_{\tau}\right|>a \quad \text { on } K_{\tau}
$$

THEOREM 3.2. Let $\left(f_{\tau}\right)_{\tau \in \theta}$ be a purely finitely additive martingale, let $a>0$, let $A=\left\{\lim \sup _{\tau \in \theta}\left|f_{\tau}\right|>a\right\}$, and let $\varepsilon>0$. Then there exists a purely finitely additive martingale $\left(g_{\tau}\right)_{\tau \in \theta}$ such that

(i) $\sup _{\tau \in \theta} \int\left|g_{\tau}\right| d \mu<\varepsilon$, and

(ii) $\mu\left(A \backslash\left\{\lim \sup _{\tau \in \theta}\left|g_{\tau}\right| \geqslant a\right\}\right)<\varepsilon$.

Furthermore, if $\left(f_{\tau}\right)_{\tau \in \theta}$ is positive $\left(f_{\tau} \geqslant 0\right.$ for all $\left.\tau \in \theta\right)$, then $\left(g_{\tau}\right)_{\tau \in \theta}$ can be chosen to be positive also.

Proof. Let $0<\gamma<\varepsilon$. By Lemma 3.2 let $\left(K_{\tau}\right)_{\tau \in \theta}$ be a fine covering, let $\sigma \in \theta$, and let $C \in \mathscr{B}_{\sigma}$ be such that $\mu\left(A \backslash \lim \sup _{\tau \in \theta} K_{\tau}\right)<\varepsilon$ and $\left|1_{C} f_{\tau}\right|>a$ on $K_{\tau}$, $\int\left|1_{C} f_{\tau}\right| d \mu\left\langle\gamma\right.$ for all $\tau \gg \sigma$. Define the martingale $\left(g_{\tau}\right)_{\tau \in \theta}$ by

$$
g_{\tau}= \begin{cases}1_{C} f_{\tau} & \text { for } \tau \gg \sigma, \\ \varepsilon\left(1_{C} f_{\rho} \mid \Re_{\tau}\right) \text { for any } \rho \gg \tau, \sigma & \text { for } \tau \gg \sigma .\end{cases}
$$


Clearly $\left(g_{\tau}\right)_{\tau \in \theta}$ is a purely finitely additive martingale. Also $\left(g_{\tau}\right)_{\tau \in \theta}$ is positive if $\left(f_{\tau}\right)_{\tau \in \theta}$ is positive. Furthermore,

$$
\sup _{\tau \in \theta} \int\left|g_{\tau}\right| d \mu=\sup _{\tau \gg \sigma} \int\left|g_{\tau}\right| d \mu=\sup _{\tau \gg \sigma} \int\left|1_{C} f_{\tau}\right| d \mu<\gamma<\varepsilon .
$$

Finally,

$$
\begin{aligned}
\left\{\limsup _{\tau \in \theta}\left|g_{\tau}\right| \geqslant a\right\} & \supseteq \limsup _{\tau \in \theta}\left\{\left|g_{\tau}\right|>a\right\} \\
& =\limsup _{\tau \in \theta}\left\{\left|1_{C} f_{\tau}\right|>a\right\} \supseteq \underset{\tau \in \theta}{\lim \sup } K_{\tau},
\end{aligned}
$$

and

$$
\mu\left(A \backslash\left\{\limsup _{\tau \in \theta}\left|g_{\tau}\right| \geqslant a\right\}\right) \leqslant \mu\left(A \backslash \lim \sup _{\tau \in \theta} K_{\tau}\right)<\varepsilon
$$

4. Dominated sums and order convergence of martingales. We first prove order convergence theorems for countably additive and purely finitely additive martingales, and then we combine these results into a general order convergence theorem.

The following proposition is used in the proof of the order convergence theorem for countably additive martingales. The proof of the proposition is elementary.

Proposition 4.1. Let $B \in \mathscr{B}$ be such that every positive function $f$ with $\int f d \mu<$ $\infty$ and every $a>b>0$ satisfies $\left\{\lim \sup _{\tau \in \theta} \mathcal{E}\left(f \mid \Re_{\tau}\right)>a>b>\mathcal{E}\left(f \mid \Re_{\infty}\right)\right\} \cap B$ $=\varnothing$. Let $g$ be a function with $\int|g| d \mu<\infty$. Then

$$
\underset{\tau \in \theta}{\lim \sup } \mathcal{E}\left(g \mid \mathscr{B}_{\tau}\right)=\mathcal{E}\left(g \mid \mathscr{B}_{\infty}\right)=\liminf _{\tau \in \theta} \mathcal{E}\left(g \mid \mathscr{B}_{\tau}\right) \text { on } B .
$$

Proof. Our proof is by contradiction. It is easy to show that $\lim \inf _{\tau \in \theta} \mathcal{E}\left(g \mid \mathscr{B}_{\tau}\right)$ $<\lim \sup _{\tau \in \theta} \mathcal{E}\left(g \mid \mathscr{B}_{\tau}\right)$. Assume $\lim \sup _{\tau \in \theta} \mathcal{E}\left(g \mid \mathscr{B}_{\tau}\right)=\mathcal{E}\left(g \mid \mathscr{B}_{\infty}\right)=$ $\lim \inf _{\tau \in \theta} \mathcal{E}\left(g \mid \Re_{\tau}\right)$ fails to hold everywhere on $B$. Then either

$$
\left\{\lim \sup _{\tau \in \theta} \mathcal{E}\left(g \mid \mathscr{B}_{\tau}\right)>\mathcal{E}\left(g \mid \mathscr{B}_{\infty}\right)\right\} \cap B \neq \varnothing
$$

or

$$
\left\{\liminf _{\tau \in \theta} \mathcal{E}\left(g \mid \Re_{\tau}\right)<\mathcal{E}\left(g \mid \Re_{\infty}\right)\right\} \cap B \neq \varnothing .
$$

By considering $g$ and $-g$, with $\lim \sup _{\tau \in \theta} \mathcal{E}\left(-g \mid \Re_{\tau}\right)=-\lim \inf _{\tau \in \theta} \mathcal{E}\left(g \mid \Re_{\tau}\right)$, we can assume that

$$
\left\{\underset{\tau \in \theta}{\lim \sup } \mathcal{E}\left(g \mid \mathscr{B}_{\tau}\right)>\mathcal{E}\left(g \mid \mathscr{B}_{\infty}\right)\right\} \cap B \neq \varnothing
$$

Let $c>d$ be such that the set

$$
C=\left\{\limsup _{\tau \in \theta} \mathcal{E}\left(g \mid \mathscr{S}_{\tau}\right)>c>d>\mathcal{E}\left(g \mid \mathscr{G}_{\infty}\right)\right\}
$$


has nonempty intersection with $B$. Let $D \in \cup_{\tau \in \theta} \mathscr{B}_{\tau}$ be such that $\mu(D)<\infty$ and $D \cap C \cap B \neq \varnothing$. Define the real numbers $a, b$, the function $h$, and $F \in \mathscr{B}_{\infty}$ by

$$
\begin{gathered}
a=c+|d|+1, \quad b=d+|d|+1, \quad h=g+(|d|+1) 1_{D}, \\
F=\left\{\limsup _{\tau \in \theta} \varepsilon\left(h \mid \mathscr{B}_{\tau}\right)>a>b \geqslant \varepsilon\left(h \mid \mathscr{B}_{\infty}\right)\right\} .
\end{gathered}
$$

Then $a>b>0, \int|h| d \mu \leqslant \int|g| d \mu+(|d|+1) \mu(D)<\infty$,

$$
\begin{aligned}
\underset{\tau \in \theta}{\lim \sup } \mathcal{E}\left(h \mid \mathscr{S}_{\tau}\right) & =\limsup _{\tau \in \theta}\left(\mathcal{E}\left(g \mid \Re_{\tau}\right)+(|d|+1) 1_{D}\right) \\
& =\left(\limsup _{\tau \in \theta} \mathcal{E}\left(g \mid \Re_{\tau}\right)\right)+(|d|+1) 1_{D}
\end{aligned}
$$

and $\mathcal{E}\left(h \mid \mathscr{B}_{\infty}\right)=\mathcal{E}\left(g \mid \mathfrak{B}_{\infty}\right)+(|d|+1) 1_{D}$; hence $F \supseteq D \cap C$. Finally, let $f=$ $\sup \left(0, \mathcal{E}\left(h \mid \Re_{\infty}\right)\right)$. Then $f$ is a positive function with $\int f d \mu<\int|h| d \mu<\infty$,

$$
\begin{aligned}
\lim \sup _{\tau \in \theta} \mathcal{E}\left(f \mid \mathscr{B}_{\tau}\right) & \geqslant \operatorname{lim\operatorname {sup}} \mathcal{E}\left(\mathcal{E}\left(h \mid \mathscr{B}_{\infty}\right) \mid \mathscr{G}_{\tau}\right) \\
& =\limsup _{\tau \in \theta} \mathcal{E}\left(h \mid \mathscr{B}_{\tau}\right),
\end{aligned}
$$

and

$$
\begin{gathered}
\left\{\lim \sup _{\tau \in \theta} \mathcal{E}\left(f \mid \mathscr{B}_{\tau}\right)>a>b \geqslant \mathcal{E}\left(f \mid \mathscr{\Re}_{\infty}\right)\right\} \cap B \\
\supseteq F \cap B \supseteq D \cap C \cap B \neq \varnothing,
\end{gathered}
$$

which is a contradiction.

THEOREM 4.1. Let the family $\left(\mathscr{B}_{\tau}\right)_{\tau \in \theta}$ possess the dominated sums property. Then every countably additive martingale $\left(\mathcal{E}\left(f \mid \mathscr{G}_{\tau}\right)\right)_{\tau \in \theta}$ is order convergent to $\mathcal{E}\left(f \mid \mathscr{\Re}_{\infty}\right)$.

Proof. Our proof is by contradiction. Assume there exists a countably additive martingale $\left(\mathcal{E}\left(g \mid \mathscr{B}_{\tau}\right)\right)_{\tau \in \theta}$ which is not order convergent to $\mathcal{E}\left(g \mid \mathscr{B}_{\infty}\right)$. Then by Proposition 4.1 there exist a positive function $f$, with $\int f d \mu<\infty$, and $a>b>0$ such that the set

$$
A=\left\{\limsup _{\tau \in \theta} \mathcal{E}\left(f \mid \mathscr{B}_{\tau}\right)>a>b \geqslant \mathcal{E}\left(f \mid \mathscr{B}_{\infty}\right)\right\}
$$

is nonempty. Let $\left(K_{\tau}\right)_{\tau \in \theta}$ be the fine covering of $A$ prescribed by Lemma 3.1. Let $\varepsilon>0$. Choose $\sigma \in \theta$ and $C \in \mathscr{B}_{\infty}$ such that

(i) $\int 1_{C} f d \mu<\varepsilon$, and

(ii) $\mathcal{E}\left(1_{c} f \mid \mathscr{B}_{\tau}\right) \geqslant a-b$ on $K_{\tau}$ for each $\tau \gg \sigma$. Let $\left(\xi_{1}, \xi_{2}, \ldots, \xi_{r}\right)$ and $\mathcal{E}=$ $\left(L_{1}, L_{2}, \ldots, L_{r}\right)$ be finite sequences of $\theta$-members and $\mathscr{B}$-members satisfying $L_{i} \in \mathscr{B}_{\xi}, L_{i} \subseteq K_{\xi}$ and $\xi_{i} \gg \sigma$ for each $i=1,2, \ldots, r$. Then for each $i=$ $1,2, \ldots, r$,

$$
\int_{L_{i}} 1_{C} f d \mu=\int_{L_{i}} \mathcal{E}\left(1_{C} f \mid \mathscr{B}_{\xi_{i}}\right) d \mu \geqslant \int_{L_{i}} a-b d \mu=(a-b) \mu\left(L_{i}\right)
$$


Summing,

$$
\begin{aligned}
\left\|\sum \mathcal{L}\right\|_{\infty} \varepsilon & \geqslant \int\left(\sum \mathcal{L}\right)\left(1_{C} f\right) d \mu=\sum_{i=1}^{r} \int_{L_{i}} 1_{C} f d \mu \\
& \geqslant(a-b)\left\|\sum \mathcal{E}\right\|_{1},
\end{aligned}
$$

and $\left(K_{\tau}\right)_{\tau \in \theta}$ fails to satisfy the dominated sums condition.

THEOREM 4.2. Let the family $\left(\mathscr{G}_{\tau}\right)_{\tau \in \theta}$ possess the dominated sums property. Then every purely finitely additive martingale is order convergent to 0 . (We denote by 0 the function which takes the value 0 everywhere.)

Proof. Our proof is by contradiction. Let $\left(f_{\tau}\right)_{\tau \in \theta}$ be a purely finitely additive martingale, and assume for some $a>0$, the set $A=\left\{\lim \sup _{\tau \in \theta}\left|f_{\tau}\right|>a\right\}$ is nonempty. Let $0<\varepsilon<\mu(A)$. Let $\left(K_{\tau}\right)_{\tau \in \theta}$ be the fine covering prescribed by Lemma 3.2. Then $\lim \sup _{\tau \in \theta} K_{\tau} \neq \varnothing$. Let $\gamma>0$. Choose $\sigma \in \theta$ and $C \in \mathscr{B}_{\sigma}$ such that for each $\tau \gg \sigma$,

(a) $\int\left|1_{c} f_{\tau}\right| d \mu<\gamma$, and

(b) $\left|1_{c} f_{\tau}\right|>a$ on $K_{\tau}$.

Let $\left(\xi_{1}, \xi_{2}, \ldots, \xi_{r}\right)$ and $\mathcal{L}=\left(L_{1}, L_{2}, \ldots, L_{r}\right)$ be finite sequences of $\theta$-members and $\mathscr{B}$-members satisfying $L_{i} \in \mathscr{B}_{\xi}, L_{i} \subseteq K_{\xi}$, and $\xi_{i} \gg \sigma$ for each $i=1,2, \ldots, r$. Choose $\rho \in \theta$ such that $\rho \gg \xi_{i}$ for all $i=1,2, \ldots, r$. Then for each $i=$ $1,2, \ldots, r$,

$$
\int_{L_{i}}\left|1_{C} f_{\rho}\right| d \mu \geqslant \int_{L_{i}}\left|1_{C} f_{\xi}\right| d \mu \geqslant \int_{L_{i}} a d \mu=a \mu\left(L_{i}\right)
$$

Summing,

$$
\begin{aligned}
\left\|\sum \mathcal{L}\right\|_{\infty} \gamma & \geqslant \int\left(\sum \mathcal{L}\right)\left|1_{C} f_{\rho}\right| d \mu=\sum_{i=1}^{r} \int_{L_{i}}\left|1_{C} f_{\rho}\right| d \mu \\
& \geqslant a\left\|\sum \mathcal{L}\right\|_{1},
\end{aligned}
$$

and $\left(K_{\tau}\right)_{\tau \in \theta}$ fails to satisfy the dominated sums condition.

We remark that Krickeberg [4], [5] has already identified the limit of any order convergent martingale of bounded variation. In fact, he has proved that every countably additive martingale $\left(\mathcal{E}\left(f \mid \mathscr{B}_{\tau}\right)\right)_{\tau \in \theta}$ satisfies

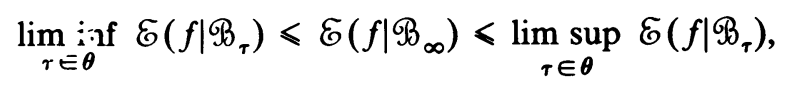

and every purely finitely additive martingale $\left(g_{\tau}\right)_{\tau \in \theta}$ satisfies

$$
\liminf _{\tau \in \theta} g_{\tau}<0 \leqslant \lim \sup _{\tau \in \theta} g_{\tau} \text {. }
$$

(See also [2].)

Proposition 4.2. Let $B \in \mathscr{B}$ be such that all martingales of bounded variation $\left(g_{\tau}\right)_{\tau \in \theta}$ satisfy $\lim \sup _{\tau \in \theta} g_{\tau}=\lim _{\inf _{\tau \in \theta}} g_{\tau}$ on $B$. Then all martingales of semibounded variation $\left(f_{\tau}\right)_{\tau \in \theta}$ satisfy $\lim \sup _{\tau \in \theta} f_{\tau}=\lim _{\inf _{\tau \in \theta}} f_{\tau}$ on $B$. 
Proof. Our proof is by contradiction. Assume $\left(f_{\tau}\right)_{\tau \in \theta}$ is a martingale of semibounded variation and $a$ is a real number such that the set

$$
A=\left\{\limsup _{\tau \in \theta} f_{\tau}>a>\liminf _{\tau \in \theta} f_{\tau}\right\} \cap B
$$

is nonempty. Assume the set $\left\{\int_{C} f_{\tau} d \mu \mid \tau \in \theta, C \in \mathscr{B}_{\tau}\right\}$ is bounded from above. (The other case is symmetric; moreover, considering the martingale $\left(-f_{\tau}\right)_{\tau \in \theta}$ reduces the other case to the present case.) Now $A \subseteq \lim \sup _{\tau \in \theta}\left\{f_{\tau}>a\right\}$ and there exist $\sigma \in \theta$ and $D \in \mathscr{B}_{\sigma}$ such that $D \subseteq\left\{f_{\sigma}>a\right\}, \mu(D)<\infty$, and $D \cap A \neq \varnothing$. Define the martingale $\left(g_{\tau}\right)_{\tau \in \theta}$ by

$$
g_{\tau}= \begin{cases}1_{D} f_{\tau} & \text { for } \tau \gg \sigma, \\ \mathcal{E}\left(1_{D} f_{\rho} \mid \mathscr{B}_{\tau}\right) \text { for any } \rho \gg \tau, \sigma & \text { for } \tau \gg \sigma .\end{cases}
$$

Then

$$
\begin{gathered}
\limsup g_{\tau}=\limsup _{\tau \in \theta} 1_{D} f_{\tau}=1_{D} \limsup f_{\tau \in \theta}, \\
\liminf _{\tau \in \theta} g_{\tau}=\liminf _{\tau \in \theta} 1_{D} f_{\tau}=1_{D} \liminf _{\tau \in \theta} f_{\tau},
\end{gathered}
$$

and

$$
\underset{\tau \in \theta}{\lim \sup } g_{\tau}>a>\liminf _{\tau \in \theta} g_{\tau} \text { on } D \cap A \subseteq B .
$$

Furthermore, for $\tau \in \theta$ and $C \in \mathscr{B}_{\tau}$,

$$
\begin{aligned}
\int_{C} g_{\tau} d \mu & =\int_{C} g_{\rho} d \mu \quad \text { for some } \rho \gg \tau, \sigma \\
& =\int g_{\rho} d \mu-\int_{E \backslash C} g_{\rho} d \mu=\int g_{\sigma} d \mu-\int_{E \backslash C} g_{\tau} d \mu>a \mu(D)-\int_{E \backslash C} g_{\tau} d \mu .
\end{aligned}
$$

Therefore $\left\{\int_{C} g_{\tau} d \mu \mid \tau \in \theta, C \in \mathscr{B}_{\tau}\right\}$, which is clearly bounded from above, is also bounded from below. Hence $\left(g_{\tau}\right)_{\tau \in \theta}$ is of bounded variation, which is a contradiction.

THEOREM 4.3. Let the family $\left(\mathscr{B}_{\tau}\right)_{\tau \in \theta}$ possess the dominated sums property. Then every martingale of semibounded variation is order convergent.

Proof. Proposition 4.2, Theorems 4.1, 4.2, and the decomposition of a martingale into countably additive and purely finitely additive parts.

We immediately recover Krickeberg's [3] order convergence theorem.

THEOREM 4.4. Let $\left(\mathscr{B}_{\tau}\right)_{\tau \in \theta}$ possess the Vitali property. Then every martingale of semibounded variation is order convergent.

5. Decompositions of $E$ in terms of order convergence of martingales. Let $B \in \mathscr{B}_{\infty}$, and let $\left(f_{\tau}\right)_{\tau \in \theta}$ be a family of functions. Whenever $\lim \inf _{\tau \in \theta} f_{\tau}=$ $\lim \sup _{\tau \in \theta} f_{\tau}$ on $B$ we say that $\left(f_{\tau}\right)_{\tau \in \theta}$ is order convergent on $B$, and if $f=$ $\lim \inf _{\tau \in \theta} f_{\tau}=\lim _{\sup _{\tau \in \theta}} f_{\tau}$ on $B$ we say that $\left(f_{\tau}\right)_{\tau \in \theta}$ is order convergent to $f$ on $B$. 
THEOREM 5.1. There exist $A_{c}, B_{c} \in \mathscr{B}_{\infty}, B_{c}=E \backslash A_{c}$, such that:

(i) there exists a positive function $f$ satisfying $\int f d \mu<\infty$ and $\lim \sup _{\tau \in \theta} \mathcal{E}\left(f \mid \Re_{\tau}\right)$ $=\infty$ on $A_{c}$

(ii) for every function $g$ with $\int|g| d \mu<\infty$, the martingale $\left(\mathcal{E}\left(g \mid \Re_{\tau}\right)\right)_{\tau \in \theta}$ is order convergent to $\mathcal{E}\left(g \mid \mathscr{B}_{\infty}\right)$ on $B_{c}$.

Proof. For each positive function $f$ with $\int f d \mu<\infty$, and for each $a>b>0$, let

$$
A(f, a, b)=\left\{\limsup _{\tau \in \theta} \mathcal{E}\left(f \mid \mathscr{B}_{\tau}\right)>a>b \geqslant \mathcal{E}\left(f \mid \mathscr{B}_{\infty}\right)\right\} \in \mathscr{B}_{\infty} .
$$

Define $A_{c} \in \mathscr{B}_{\infty}$ to be the (essential) union of the family of all sets $A(f, a, b)$. Let $N$ be the set of positive integers. There exists a countable subfamily, $\left\{A\left(f_{i}, a_{i}, b_{i}\right) \mid i\right.$ $\in N$ \}, whose union is $A_{c}$. Applying Theorem 3.1 for each $i, j \in N, i<j$, let $C_{i, j} \in \mathscr{B}_{\infty}$ be such that

$$
\int 1_{C_{i, j}} f_{i} d \mu \leqslant \frac{1}{j 2^{i+j}}
$$

and

$$
\underset{\tau \in \theta}{\lim \sup } \mathcal{E}\left(1_{C_{i, j}} f_{i} \mid \Re_{\tau}\right) \geqslant a_{i}-b_{i} \text { on } A\left(f_{i}, a_{i}, b_{i}\right)
$$

Define

$$
f=\sum_{\substack{i, j \in N \\ i<j}} j 1_{c_{i, j}} f_{i}
$$

Then $f$ is positive and

$$
\int f d \mu=\sum_{\substack{i, j \in N \\ i<j}} j \int 1_{C_{i, j}} f_{i} d \mu<\sum_{\substack{i, j \in N \\ i<j}} \frac{1}{2^{i+j}}<1 .
$$

Furthermore, for each $i, j \in N, i \leqslant j$,

$$
\begin{aligned}
\limsup _{\tau \in \theta} E\left(f \mid \mathscr{B}_{\tau}\right) & \geqslant \underset{\tau \in \theta}{\lim \sup } \mathcal{E}\left(j 1_{C_{i, j}} f_{i} \mid \mathscr{G}_{\tau}\right) \\
& \geqslant j\left(a_{i}-b_{i}\right) \quad \text { on } A\left(f_{i}, a_{i}, b_{i}\right) ;
\end{aligned}
$$

hence

$$
\underset{\tau \in \theta}{\lim \sup } \mathcal{E}\left(f \mid \mathscr{\Im}_{\tau}\right)=\infty \quad \text { on } A_{c}=\bigcup_{i \in N} A\left(f_{i}, a_{i}, b_{i}\right) .
$$

Define $B_{c}=E \backslash A_{c}$. Proposition 4.1 completes the proof.

In the proof of our next theorem we make use of the Jordan decomposition of martingales [3]: Every martingale of bounded variation can be expressed as the difference of two positive martingales of bounded variation. It is easy to show that every purely finitely additive martingale can be expressed as the difference of two positive purely finitely additive martingales.

We also make use of the following result, which is a consequence of a corresponding result for purely finitely additive set functions, or which can be established with an elementary argument using the characterization of purely finitely 
additive martingales given in $\S 3$. Let $N$ be the set of positive integers, let $\left\{\left(f_{i, \tau}\right)_{\tau \in \theta} \mid i\right.$ $\in N\}$ be a family of purely finitely additive martingales satisfying $\sum_{i \in N} \sup _{\tau \in \theta} \int\left|f_{i, \tau}\right| d \mu<\infty$, and let $f_{\tau}=\sum_{i \in N} f_{i, \tau}$ for each $\tau \in \theta$; then $\left(f_{\tau}\right)_{\tau \in \theta}$ is a purely finitely additive martingale.

TheOREM 5.2. There exist $A_{p}, B_{p} \in \mathscr{B}_{\infty}, B_{p}=E \backslash A_{p}$, such that:

(i) there exists a positive purely finitely additive martingale $\left(f_{\tau}\right)_{\tau \in \theta}$ satisfying $\lim \sup _{\tau \in \theta} f_{\tau}=\infty$ on $A_{p}$;

(ii) every purely finitely additive martingale is order convergent to 0 on $B_{p}$.

Proof. For each positive purely finitely additive martingale $\Phi=\left(g_{\tau}\right)_{\tau \in \theta}$ and for each $a>0$ let $A(\Phi, a)=\left\{\lim \sup _{\tau \in \theta} \Phi>a\right\}$. Define $A_{p}$ to be the (essential) union of the family of all sets $A(\Phi, a)$. Let $N$ be the set of positive integers. There exists a countable subfamily, $\left\{A\left(\Phi_{i}, a_{i}\right) \mid i \in N\right\}$, whose union is $A_{p}$. Applying Theorem 3.2 for each $i, j \in N, i \leqslant j$, let $\left(f_{i, j, \tau}\right)_{\tau \in \theta}$ be a positive purely finitely additive martingale such that

$$
\sup _{\tau \in \theta} \int f_{i, j, \tau} d \mu<\frac{1}{j 2^{i+j}}
$$

and

$$
\mu\left(A\left(\Phi_{i}, a_{i}\right) \backslash\left\{\limsup _{\tau \in \theta} f_{i, j, \tau} \geqslant a_{i}\right\}\right)<\frac{1}{j 2^{i+j}} .
$$

Define the martingale $\left(f_{\tau}\right)_{\tau \in \theta}$ by

$$
f_{\tau}=\sum_{\substack{i, j \in N \\ i<j}} j f_{i, j, \tau} \quad \text { for } \tau \in \theta .
$$

Then $\left(f_{\tau}\right)_{\tau \in \theta}$ is a positive purely finitely additive martingale. Furthermore, for each $i, j \in N, i \leqslant j$,

$$
\lim \sup _{\tau \in \theta} f_{\tau} \geqslant \limsup _{\tau \in \theta} j f_{i, j, \tau} \geqslant j a_{i} \text { on } A\left(\Phi_{i}, a_{i}\right)
$$

except for a set of $\mu$-measure $1 / j 2^{i+j}$; hence

$$
\underset{\tau \in \theta}{\lim \sup } f_{\tau}=\infty \quad \text { on } A_{p}=\bigcup_{i \in N} A\left(\Phi_{i}, a_{i}\right)
$$

Let $B_{p}=E \backslash A_{p}$. By the definition of $A_{p}$, all positive purely finitely additive martingales are order convergent to 0 on $B_{p}$. The Jordan decomposition of martingales completes the proof.

We now combine Theorems 5.1 and 5.2.

TheOREM 5.3. There exist $A, B \in \mathscr{B}_{\infty}, B=E \backslash A$, such that:

(i) there exists a positive martingale of bounded variation $\left(f_{\tau}\right)_{\tau \in \theta}$ satisfying $\lim \sup _{\tau \in \theta} f_{\tau}=\infty$ on $A$;

(ii) every martingale of semibounded variation is order convergent on $B$.

Proof. Theorems 5.1 and 5.2 with $A=A_{c} \cup A_{p}$, Proposition 4.2, and the decomposition of a martingale into countably additive and purely finitely additive parts. 
Corollary 5.1. Assume every positive martingale of bounded variation $\left(f_{\tau}\right)_{\tau \in \theta}$ satisfies $\lim \sup _{\tau \in \theta} f_{\tau}<\infty$ on $E$. Then every martingale of semibounded variation is order convergent.

Similar corollaries hold for countably additive and purely finitely additive martingales.

\section{REFERENCES}

1. H. Hahn and A. Rosenthal, Sét functions, Univ. of New Mexico Press, Albuquerque, N. M., 1948.

2. C. A. Hayes and C. Y. Pauc, Derivation and martingales, Springer-Verlag, Berlin, 1970.

3. K. Krickeberg, Convergence of martingales with a directed index set, Trans. Amer. Math. Soc. 83 (1956), 313-337.

4. __ Stochastische Konvergenz von Semimartingalen, Math. Z. 66 (1957), 470-486.

5. __ Bemerkungen zur stochastischen Konvergenz, Bull. Soc. Math. Grèce 5 (1964), Fasc. 1, 81-92.

6. K. Yosida and E. Hewitt, Finitely additive measures, Trans. Amer. Math. Soc. 72 (1952), 46-66.

Department of Mathematics, Wayne State University, Detroit, Michigan 48202

Current address: TRW Defense and Space Systems Group, One Space Park, Redondo Beach, California 90278 